\title{
Local Stability of Curzon-Ahlborn Cycle with Non-Linear Heat Transfer for Maximum Power Output Regime
}

\author{
Delfino Ladino-Luna, Pedro Portillo-Díaz, Ricardo T. Páez-Hernández \\ Universidad Autónoma Metropolitana-Atzcapotzalco, Física de Procesos Irreversibles, Cd. México, México \\ Email: dll@correo.azc.uam.mx,phrt@correo.azc.uam.mx
}

Received May 7, 2013; revised June 9, 2013; accepted July 4, 2013

Copyright (c) 2013 Delfino Ladino-Luna et al. This is an open access article distributed under the Creative Commons Attribution License, which permits unrestricted use, distribution, and reproduction in any medium, provided the original work is properly cited.

\begin{abstract}
The study of local stability of thermal engines modeled as an endoreversible Curzon and Ahlborn cycle is shown. It is assumed a non-linear heat transfer for heat fluxes in the system (engine + environments). A semisum of two expressions of the efficiency found in the literature of finite time thermodynamics for the maximum power output regime is considered in order to make the analysis. Expression of variables for local stability and power output is found even graphic results for important parameters in the analysis of stability, and a phase plane portrait is shown.
\end{abstract}

Keywords: Local Stability; Thermal Engines; Non-Linear Heat Transfer

\section{Introduction}

As it is known the limits in the performance of thermal engines in the Classical Equilibrium Thermodynamics context correspond to reversible processes [1-4]. This situation represents a very hard obstacle in the analysis of thermal engines and leads to non adequate values for variables of processes whose values far from to the experimental values were reported in the literature. These limits have been partially overcome by helping of the named Finite Time Thermodynamics [5-7]. In order to analyze the performance of thermal engines, many papers in this context have considered that the heat flux between the system and its environs is made by Newton heat transfer law [5-14], for the named Curzon and Ahlborn engine [7]. Nevertheless a more real model has to consider all possibilities of heat transfer. Thus, some authors have used particularly the Dulong and Petit heat transfer law because it allows a better model than the Newton heat transfer model is [15-18]. In [15-17] numerical results appear near to the experimental values reported in the literature for power plants working at maximum power output, and in $[17,18]$ for nuclear plants working at maximum ecological function [14].

It is important to point out that all of the above-cited papers have been focused on the thermodynamics properties of the system, through an objective function to analyze the performance of thermal engines, and only the steady state has been analyzed.
Nevertheless, other authors have analyzed the intrinsic properties of the systems as the response to a perturbation on the steady state of important quantities for the performance of thermal engines $[19,20]$. More recently the local stability of thermal engines has been made considering Newton heat transfer [21-24] and Stefan-Boltzmann heat transfer [25], besides it has been made considering a working substance different to ideal gas [26].

In the present paper, we consider a heat transfer like the Dulong-Petit heat transfer law in order to make the analysis of a thermal engine for local stability. The important quantities in the performance of the thermal engine are found by the heat transfer cited, as the expression of power output and the dynamic equations for an endoreversible Curzon and Ahlborn engine. We assume for simplicity an expression of efficiency as a semi-sum of two expressions found by two different authors [1618], which contains the same necessary parameters of the present work, including a comparison by plotting of them. To make this paper self-contained, a review of some wellknown results on the Carnot, and Curzon and Ahlborn engines concerning to steady state variables is also included.

\section{Properties of the Steady States}

\subsection{Steady States Variables}

Let us consider a system which consists of two reservoirs at temperatures $T_{1}$ (hot reservoir) and $T_{2}$ (cold reser- 
voir), which are related as $T_{1}>T_{2}$; and the thermal engine working at temperatures $x$ and $y$, which are related as $x>y$. There is a resistance for the heat flow between the thermal engine and its reservoirs with a heat conductance denoted by $\alpha$, as is shown in Figure 1. In case of Carnot engine, $T_{1} \equiv x$ and $y \equiv T_{2}$, and in case of Curzon and Ahlborn engine the temperatures are related by $T_{1}>x>y>T_{2}$. The engine produces the work $W$. The heat $Q_{1}$ flows from the hot reservoir to the engine and the heat $Q_{2}$ flows from the engine to the cold reservoir, assuming a constant thermal conductance by the given parameter $\alpha$ in both fluxes.

According to the first and second laws of thermodynamics, for Carnot engine the heats exchanged in the system $Q_{1}$ and $Q_{2}$ are given by

$$
Q_{1}=\frac{x}{x-y} W
$$

and

$$
Q_{2}=\frac{y}{x-y} W .
$$

For the endoreversible Curzon and Ahlborn engine we can consider an engine working in steady state, so the temperatures are now $\bar{x}$ and $\bar{y}$ with $T_{1}>\bar{x}>\bar{y}>T_{2}$ [20]. Here, and thereafter, the variables with over-bars represent steady state quantities. The endoreversible hypothesis is now,

$$
\frac{\bar{J}_{1}}{T_{1}}=\frac{\bar{J}_{2}}{T_{2}}
$$

which asserts that an engine working between two reservoirs at temperatures $\bar{x}$ and $\bar{y}$ behaves as a Carnot

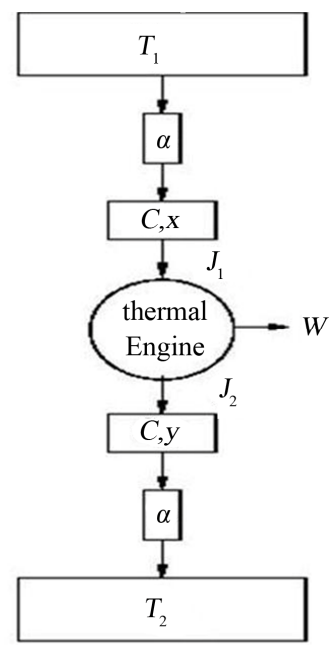

Figure 1. Thermal engine working between both $x$ and $y$ temperatures. The reservoirs are at temperatures $T_{1}$ and $T_{2}$. The fluxes to the engine and from the engine are respectively $J_{1}$ and $J_{2}$. engine, despite the fact that it works in finite-time cycles [10]. $\bar{J}_{1}$ and $\bar{J}_{2}$ are the steady state heat flows from the $\bar{x}$ to the engine and from the engine to $\bar{y}$, respectively, and because the Curzon and Ahlborn engine is usually supposed working in steady state, the fluxes before mentioned are

$$
\bar{J}_{1}=\alpha\left(T_{1}-\bar{x}\right)
$$

and

$$
\bar{J}_{2}=\alpha\left(\bar{y}-T_{2}\right)
$$

This means that

$$
\bar{J}_{1}=\frac{\bar{x}}{\bar{x}-\bar{y}} \bar{P}
$$

and

$$
\bar{J}_{2}=\frac{\bar{y}}{\bar{x}-\bar{y}} \bar{P}
$$

$\bar{P}$ is the power output in steady state. The efficiency in steady state for this internally reversible Curzon and Ahlborn engine is written as

$$
\bar{\eta}=1-\frac{\bar{y}}{\bar{x}}
$$

and by using (4b) we can write

$$
\bar{\eta}=1-\frac{\bar{J}_{2}}{\bar{J}_{1}}=\bar{W} \bar{P} \text { or } \bar{\eta}=\frac{\bar{P}}{\bar{J}_{1}},
$$

and it follows that

$$
\bar{x}=\frac{T_{1}}{2}\left(1+\frac{T_{2} / T_{1}}{1-\bar{\eta}}\right)
$$

and

$$
\bar{y}=\frac{T_{1}}{2}(1-\bar{\eta})\left(1+\frac{T_{2} / T_{1}}{1-\bar{\eta}}\right) .
$$

\subsection{Effect of a Non-Linear Heat Transfer Law}

Consider now a heat transfer law as,

$$
\frac{\mathrm{d} Q}{\mathrm{~d} t}=\alpha\left(T_{0}-T\right)^{k},
$$

which contains as a particular case the Dulong and Petit heat transfer law, where $\mathrm{d} Q / \mathrm{d} t$ is the rate of heat transfer, $\alpha$ is the thermal conductance, $T_{0}$ is the temperature of environs, $T$ is the temperature of the body and $k$ is a parameter as $k>1$, which in case of Dulong and Petit heat transfer is as $1.1 \leq k \leq 1.6 \quad[27,28]$.

Heat fluxes $\bar{J}_{1}, \bar{J}_{2}$ can be written now as

$$
\bar{J}_{1}=\alpha\left(T_{1}-\bar{x}\right)^{k} \text { and } \bar{J}_{2}=\alpha\left(\bar{y}-T_{2}\right)^{k}, k>1 \text {, }
$$

and (6) with (9) permits 


$$
\bar{x}=\bar{y} \frac{\left(T_{1}-\bar{x}\right)^{k}}{\left(\bar{y}-T_{2}\right)^{k}},
$$

and then,

$$
\bar{x}=T_{1} \frac{(1-\bar{\eta})^{1 / k}+\tau}{(1-\bar{\eta})+(1-\bar{\eta})^{1 / k}},
$$

and,

$$
\bar{y}=(1-\bar{\eta}) T_{1} \frac{(1-\bar{\eta})^{1 / k}+\tau}{(1-\bar{\eta})+(1-\bar{\eta})^{1 / k}},
$$

where has been definite $\tau=T_{2} / T_{1}$.

On other hand, the Curzon and Ahlborn engine gives more realistic values of efficiency with this heat transfer than it gives with the Newton heat transfer, in case of maximum power output regime [15]. Results in [14,15] are compared with different reported values of power plants. More recently, at maximum power output regime and at maximum ecological function regime, assuming $k=5 / 4$, analytical approximated expressions for the efficiency were found as $[16,18]$,

$$
\eta_{O P D P}=1-\frac{1-\tau+\sqrt{\tau^{2}+98 \tau+1}}{10}
$$

and

$$
\eta_{\text {OEDP }}=1-\frac{1-\tau+\sqrt{649 \tau^{2}+646 \tau+1}}{36} .
$$

Besides, by a variational approach, the efficiencies in the maximum power output regime and in the ecological function regime were obtained respectively [17] as,

$$
\eta_{M P}^{(\alpha)}=1+\frac{\tau}{8}-\frac{1}{8} \sqrt{2 \tau(160+\tau)},
$$

and

$$
\eta_{M E}^{(\alpha)}=1+\frac{\tau}{8}-\frac{1}{8} \sqrt{\tau(40+41 \tau)} .
$$

Analyzing the results of several studies in the literature of finite time thermodynamics, it is found that the efficiency of Curzon and Ahlborn cycle $\eta_{C A N}$, named Curzon-Ahlborn-Novikov efficiency, is adequate for conventional plants, and the named ecological efficiency $\eta_{E}$ is adequate for modern plants (including nuclear plants). So, we consider hereafter a thermal engine in the maximum power output regime.

Comparing the efficiencies in (13a) and (14a) the following property is found,

$$
\eta_{O P D P} \leq \eta_{O B S} \leq \eta_{M P}^{(\alpha)},
$$

and the semisum $\eta_{S} \equiv 1 / 2\left(\eta_{O P D P}+\eta_{M P}^{(\alpha)}\right)$ is closer than the efficiencies in (15) to experimental efficiencies.

Table 1 shows a comparison between efficiencies in
(15), for the case of some conventional power plants working in a maximum power output regime, reported in $[16,17]$. Even more, the difference between the eficiencies (13a) and (14a) is shown in Figure 2. As can be seen, most of the numerical values of half the sum of the above cited efficiencies are an approximated constant ratio of Curzon and Ahlborn efficiency as,

$$
1 / 2\left(\eta_{O P D P}+\eta_{M P}^{(\alpha)}\right) \approx 0.88 \eta_{C A N},
$$

where the Curzon-Ahlborn-Novikov efficiency is $\eta_{C A N}=1-\sqrt{\tau}$. Besides, if the Carnot efficiency $\eta_{C}$ is considered, a numerical factor of the semisum appears also as an approximated constant ratio of this efficiency. It can be verified that the semisum $\eta_{S} \equiv 1 / 2\left(\eta_{O P D P}+\eta_{M P}^{(\alpha)}\right)$ is related with the Carnot efficiency as,

$$
1 / 2\left(\eta_{O P D P}+\eta_{M P}^{(\alpha)}\right) \approx 0.55 \eta_{C} .
$$

So, in order to analyze the local stability for a Curzon and Ahlborn engine it can be assumed the previous value for the efficiency, when the Dulong and Petit heat transfer law is considered. Figure $\mathbf{3}$ shows the difference of efficiencies in (17) as function of the parameter $\tau$, where it can be appreciated that the difference goes to zero, when $\tau \rightarrow 1$.

Using the linear approximation $(1-a)^{b} \approx 1-b a$, in

Table 1. Efficiency at maximum power output regime.

\begin{tabular}{cccccc}
\hline Power plant & $T_{2}$ & $T_{1}$ & $\eta_{M P}^{(\alpha)}$ & $\eta_{O P D P}$ & $\eta_{O B S}$ \\
\cline { 1 - 4 } $\begin{array}{c}\text { Steam power plant, West } \\
\text { Thurrock, } U \text { K }\end{array}$ & 298 & 838 & 0.37625 & 0.33577 & 0.360 \\
$\begin{array}{c}\text { Geothermal steam plant, } \\
\text { Lardarello, Italy }\end{array}$ & 353 & 523 & 0.16198 & 0.14530 & 0.160 \\
$\begin{array}{c}\text { Steam power plant, USA } \\
\text { Combined cycle plant } \\
\text { (steam-mercury), USA }\end{array}$ & 298 & 923 & 0.40380 & 0.36006 & 0.400 \\
$\begin{array}{c}\text { Central steam power }(U K \\
\text { 1936-1940) }\end{array}$ & 298 & 698 & 0.32089 & 0.28678 & 0.280 \\
\hline
\end{tabular}

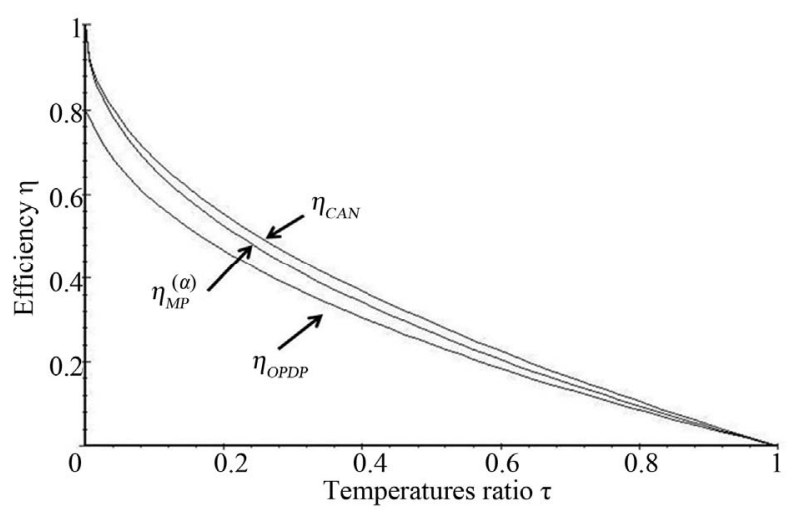

Figure 2. Graphic comparison of the efficiencies $\eta_{\text {OPDP }}, \eta_{M P}^{(\alpha)}, \eta_{C A N}$. 


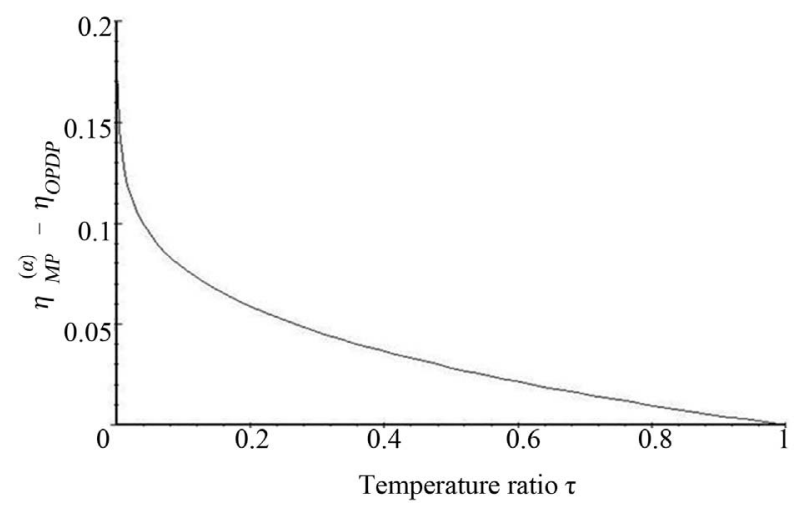

Figure 3. Behavior of the difference between the efficiencies $\eta_{M P}^{(\alpha)}$ and $\eta_{O P D P}$ as function of $\tau$.

case of $|a|<1$, and with (17) can be obtained the approximate expression for the variables in steady state as,

$$
\bar{x}=\frac{T_{1}}{2} \cdot \frac{9 T_{1}+31 T_{2}}{9 T_{1}+11 T_{2}},
$$

and

$$
\bar{y}=0.225 T_{1}+0.775 T_{2} ;
$$

and solving for $T_{1}$ and $T_{2}$ we obtain,

$$
T_{1}=\frac{22 \overline{x y}}{31 \bar{y}-9 \bar{x}}
$$

and

$$
T_{2}=4 \bar{y} \frac{9 \bar{x}-10 \bar{y}}{18 \bar{x}-31 \bar{y}} .
$$

Thus, the power output can be written as,

$$
\bar{P}=\bar{J}_{1} \bar{\eta}=\left(153.45 \bar{y}^{2}-30.6 \bar{x}-9 \bar{x}^{2}\right) \frac{\alpha(\bar{x}-\bar{y})}{\bar{x}(9 \bar{x}-31 \bar{y})} \text {. }
$$

\subsection{The Local Stability of the Curzon and Ahlborn Engine}

In order to make the analysis of local stability for an endoreversible Curzon and Ahlborn engine, we follow the procedure developed in [21], obtaining a system of coupled differential equations to model the rate of change of intermediate temperature. Let us assume the temperatures $x$ and $y$ as the corresponding to macroscopic objects with the heat capacity $\mathrm{C}$, and two differential equations for $x$ and $y$ as in [21],

$$
\frac{\mathrm{d} x}{\mathrm{~d} t}=\frac{1}{C}\left[\alpha\left(T_{1}-x\right)-J_{1}\right]
$$

and

$$
\frac{\mathrm{d} y}{\mathrm{~d} t}=\frac{1}{C}\left[J_{2}-\alpha\left(y-T_{2}\right)\right],
$$

which are cancelled when $x, y, J_{1}$ and $J_{2}$ take their steady state values. Because the assumption of endoreversibility, the heat flux from $x$ to the working fluid is $J_{1}$ and the heat flux from the thermal engine to $y$ is $J_{2}$, so these fluxes can be written as

$$
J_{1}=\frac{x}{x-y} P
$$

and

$$
J_{2}=\frac{y}{x-y} P .
$$

It is reasonable to assume that the power output from the Curzon and Ahlborn engine is related to temperatures $x$ and $y$ as the power output at steady state $\bar{P}$ depends on $\bar{x}$ and $\bar{y}$ in the maximum power output regime, then we have,

$$
P=\left(153.45 y^{2}-30.6 x-9 x^{2}\right) \frac{\alpha(x-y)}{x(9 x-31 y)} .
$$

Substituting (22) and (23) into (21) we obtain the coupled differential equations for temperatures $x$ and $y$ of a Curzon and Ahlborn engine performing in the maximum power output regime,

$$
\frac{\mathrm{d} x}{\mathrm{~d} t}=\frac{\alpha}{2 C} \cdot \frac{620 T_{1} y-180 T_{1} x-1232 x y+3069 y^{2}}{31 y-9 x},
$$

and

$$
\frac{\mathrm{d} y}{\mathrm{~d} t}=\frac{\alpha}{2 C} \times \frac{360 y x^{2}-8 y^{2} x-3069 y^{3}+620 x T_{2} y-180 T_{2} x^{2}}{x(31 y-9 x)} .
$$

To analyze the system's stability near to steady state we define two adequate functions. The differential equations in the maximum power output regime (21) are defined as the functions $f(x, y)$ and $g(x, y)$, so (24) can be written as the coupled equations from which the analysis of stability can be made,

$$
f(x, y)=\frac{\alpha}{2 C} \cdot \frac{620 T_{1} y-180 T_{1} x-1232 x y+3069 y^{2}}{31 y-9 x}
$$

and

$$
g(x, y)=\frac{\alpha}{2 C} \cdot \frac{360 y x^{2}-8 y^{2} x-3069 y^{3}+620 x T_{2} y-180 T_{2} x^{2}}{x(31 y-9 x)} .
$$

\section{Linearization and Stability Analysis}

In order to establish the consequences of a non-linear heat transfer in a thermal engine working in the maximum power output regime; we need to find the relaxation times for the corresponding eigenvectors in the stability analysis [21-25]. Moreover, if both eigenvalues are negative real numbers, perturbations decay exponentially. In 
this case it is possible to identify the characteristic time scales for each eigendirections as,

$$
t_{1}=1 /\left|\lambda_{1}\right|
$$

and

$$
t_{2}=1 /\left|\lambda_{2}\right|
$$

where $\lambda_{1}, \lambda_{2}$ are the corresponding eigenvectors. In the present case with the heat transfer law assumed we obtain the following results for the derivative of definite functions (25):

$$
\begin{aligned}
\left.f_{x}\right|_{\bar{x}, \bar{y}}= & \alpha \frac{-528.55(9+31000 \tau)^{2}(9+11 \tau)^{2}}{C\left(891+8.65 \times 10^{6} \tau+1.057 \times 10^{7} \tau^{2}\right)}, \\
\left.f_{y}\right|_{\bar{x}, \bar{y}}= & \frac{4.95 \alpha}{C\left(891+8.65 \times 10^{6} \tau+1.057 \times 10^{7} \tau^{2}\right)} \\
& \times\left(1.7983 \times 10^{6}+1.5415 \times 10^{10} \tau+7.478 \times 10^{13} \tau^{2},\right. \\
& \left.+1.83 \times 10^{14} \tau^{3}+1.1175 \times 10^{14} \tau^{4}\right) \\
\left.g_{x}\right|_{\bar{x}, \bar{y}}=\alpha & \frac{(198)\left(1175 \times 10^{14}\right)(9 / 40+775 \tau)^{2}}{C\left(891+8.65 \times 10^{6} \tau+1.057 \times 10^{7} \tau^{2}\right)^{2}} \\
\times & \frac{(9+11 \tau)^{2}\left(\tau^{2}+1.6356 \tau+0.66884\right) \tau^{2}}{(9+31 \tau)^{2}}, \\
\left.g_{y}\right|_{\bar{x}, \bar{y}}= & \alpha \frac{(28 \mathrm{a})}{C\left(891+8.65 \times 10^{6} \tau+1.057 \times 10^{7} \tau^{2}\right)^{2}} \\
& \times \frac{\left(\tau^{2}+1.6356 \tau+0.66884\right) \tau^{2}}{(9+31 \tau)^{2}} .
\end{aligned}
$$

Substituting into the characteristic equation [22-26], and taking as an example $\tau=0.5$, we obtain the eigenvalues,

$$
\left\{\begin{array}{l}
\lambda_{1}=1.0427 \times 10^{7} \\
\lambda_{2}=-5202.6
\end{array}\right.
$$

and their respective eigenvectors,

$$
\left\{\begin{array}{l}
\boldsymbol{u}_{1}=\left(-4.7452 \times 10^{-7},-0.99956\right) \\
\boldsymbol{u}_{2}=\left(-9.9994 \times 10^{-6}, 1.0509 \times 10^{-2}\right) .
\end{array}\right.
$$

Because both of the eigenvalues are real values, we can conclude that the fixed point is stable. Equations (17) and (20), respectively, determine the steady-state efficiency, $\bar{\eta}$, and the steady state power output, $\bar{P}$, as functions of $\tau=T_{2} / T_{1}$ for an endoreversible Curzon and Ahlborn engine working in a maximum-power-like regime. It is straightforward shows that both $\bar{\eta}$ and $\bar{P}$ are decreasing function of $\tau$ parameter, as is shown in Figure 4.

Both eigenvectors, $\lambda_{1}$ and $\lambda_{2}$ are function of $\tau$ and consequently relaxation times also are. There is an interval of values for relaxation times $t_{1}$ and $t_{2}$ in which they are monotone-function of $\tau$, and in the values $0 \leq \tau \leq 1$ it is, as we can appreciate in Figure 5. Finally, Figure 6 shows how all the trajectories slowly approach the origin in a tangential direction, so we can conclude that the origin is a stable point

\section{Concluding Remarks}

The present work was focused on the analysis of consequences in the stability of thermal engine when a non-linear heat transfer law is assumed. Graphic analysis shows that the engine working in these conditions is near to the steady state as it is shown in Figures $\mathbf{5}$ and 6. Combining two expressions for efficiency as a middle of them near to experimental results was an adequate decision because it permits us to have an expression as fraction of Carnot's efficiency. A comparison with other results in the literature is necessary. It is also necessary to point out that in (23) and (24) some terms in these expressions were ap-

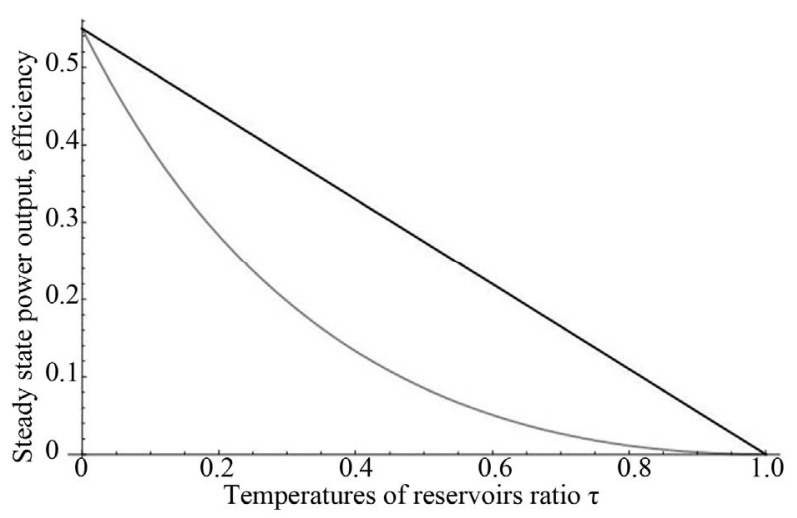

Figure 4. Steady state power output and efficiency as function of $\tau$.

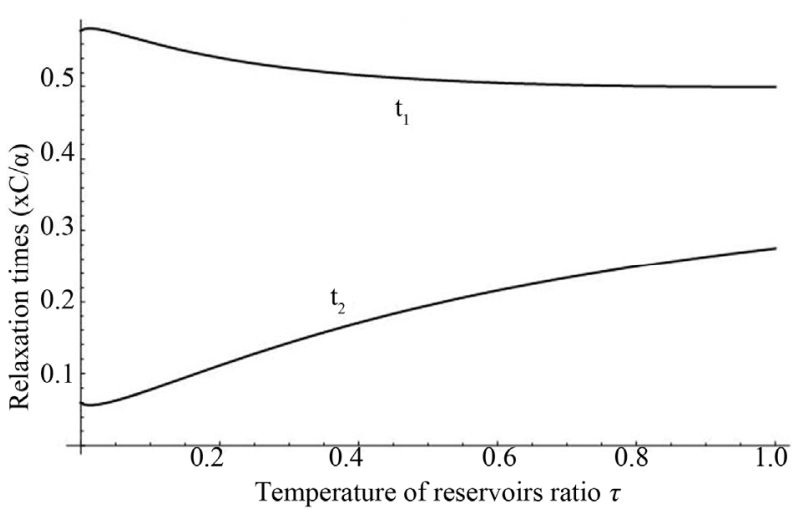

Figure 5. Relaxation times $t_{1}$ and $t_{2}$, in units of $C / \alpha$ vs $\tau$. 


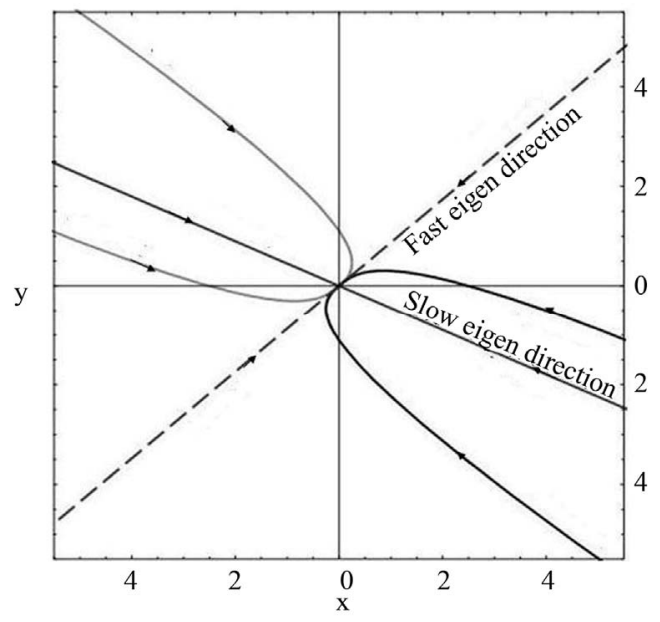

Figure 6. Qualitative phase portrait of $x(t)$ and $y(t)$ for a Curzon-Ahlborn cycle using the efficiency in (17).

proximated without losing the objective of numerical calculation.

\section{Acknowledgements}

Authors thank the partial support of CONACYT (México) by SNI program.

\section{REFERENCES}

[1] S. Carnot, "Réflexions sur la Puissance Motrice du feu et sur les Machines Propres à Développer Cette Puissanc," Spanish Translation, IPN Press, México, 1998.

[2] R. Clausius, “Théorie Mécanique de la Chaleur, 1ére Partie,” Lacroix, 1868.

[3] E. Fermi, "Thermodynamics,” Columbia University Press, New York, 1936.

[4] R. C. Tolman and P. C. Fine, Reviews of Modern Physics, Vol. 20, 1948, pp. 51-77. doi:10.1103/RevModPhys.20.51

[5] I. I. Novikov, Journal of Nuclear Energy II, Vol. 7, 1958, pp. 125-128.

[6] P. Chambadal, "Les Centrales Nucleares,” Armand Colin, 1957.

[7] F. L. Curzon and B. Ahlborn, American Journal of Physics, Vol. 43, 1975, pp. 22-24. doi:10.1119/1.10023

[8] D. Gutkowics-Krusin, I. Procaccia and J. Ross, Journal of Chemical Physics, Vol. 69, 1978, pp. 3898-3906. doi:10.1063/1.437127

[9] M. Rubin, Physical Review A, Vol. 19, 1979, pp. 12721276. doi:10.1103/PhysRevA.19.1272
[10] M. Rubin, Physical Review A, Vol. 19, 1979, pp. 1277 1289. doi:10.1103/PhysRevA.19.1277

[11] P. Salamon, A. Nitzan, B. Andresen and R. S. Berry, Physical Review A, Vol. 21, 1980, pp. 2115-2129. doi:10.1103/PhysRevA.21.2115

[12] J. L. Torres, Revista Mexicana de Física, Vol. 34, 1988, pp. $18-24$.

[13] F. Angulo-Brown, Revista Mexicana de Física, Vol. 37, 1991, pp. 87-96.

[14] F. Angulo-Brown, Journal of Applied Physics, Vol. 69, 1991, pp. 7465-7469. doi:10.1063/1.347562

[15] F. Angulo-Brown and R. T. Páez-Hernández, Journal of Applied Physics, Vol. 74, 1993, pp. 2216-2219. doi:10.1063/1.354728

[16] D. Ladino-Luna, Revista Mexicana de Física, Vol. 48, 2003, pp. 87-91.

[17] L. A. Arias-Hernández, G. Ares and F. Angulo-Brown, Open Systems \& Information Dynamics, Vol. 10, 2003, pp. 351-375. doi:10.1023/B:OPSY.0000009556.27759.11

[18] D. Ladino-Luna, Journal of the Energy Institute, Vol. 81, 2008, pp. 114-117. doi:10.1179/174602208X301961

[19] A. Lifschitz and E. Hameiri, Physical Fluids A, Vol. 3, 1991, pp. 2644-2651. doi:10.1063/1.858153

[20] G. Gallavotti, "New Methods in Noequilibrium Gases and Fluids,” arXiv:chao-dyn/9610018v1 [accesed 30.10.1996].

[21] M. Santillán, G. Maya and F. Angulo-Brown, Journal of Physics D, Vol. 34, 2001, pp. 2068-2072. doi:10.1088/0022-3727/34/13/318

[22] R. T. Páez-Hernández, F. Angulo-Brown and M. Santillán, The Journal of Non-Equilibrium Thermodynamics, Vol. 31, 2006, pp. 173-188.

[23] Y. Huang and D. Sun, The Journal of Non-Equilibrium Thermodynamics, Vol. 33, 2008, pp. 61-74.

[24] Y. Huang, D. Sun and Y. Kang, Journal of Applied Physics, Vol. 102, 2007, pp. 349051-349056. doi:10.1063/1.2767622

[25] L. Guzmán-Vargas, I. Reyes-Ramírez and N. Sánchez, Journal of Physics D: Applied Physics, Vol. 38, 2005, pp. 1282-1295. doi:10.1088/0022-3727/38/8/028

[26] R. T. Páez-Hernández, D. Ladino-Luna and P. PortilloDíaz, Physica A, Vol. 390, 2011, pp. 3275-3282. doi:10.1016/j.physa.2011.05.019

[27] L. W. Taylor, "Manual of Advanced Undergraduate Experiments in Physics,” Addison-Wesley, 1959, p. 115.

[28] M. Nelson and P. Parker, “Advanced Level Physics,” 4th Edition, Heinemann, New Orleans, 1977, p. 194. 